\title{
ON MONOTONE VS. NONMONOTONE INDUCTION
}

\author{
BY LEO A. HARRINGTON AND ALEXANDER S. KECHRIS
}

Communicated by S. Feferman, May 11, 1976

1. Introduction. For definitions and notation in what follows, see [4] and [5]. If $A$ is an infinite set and $\varphi\left(y_{1} \cdots y_{n}, R, Y_{1} \cdots Y_{m}\right)=\varphi(\bar{y}, R, \bar{Y})$ is a second order relation on $A$, we call $\varphi$ operative if $R$ is $n$-ary. For such a $\varphi$ let

$$
I_{\varphi}^{\xi}=\bigcup_{\eta<\xi} I_{\varphi}^{\eta} U\left\{(\bar{y}, \bar{Y}): \varphi\left(\bar{y},\left\{\bar{y}:(\bar{y}, \bar{Y}) \in \bigcup_{\eta<\xi} I_{\varphi}^{\eta}\right\}, \bar{Y}\right)\right\} \quad \text { and } \quad I_{\varphi}=\bigcup_{\xi} I_{\varphi}^{\xi} \text {. }
$$

If $F$ is a collection of second order relations (for simplicity collection of operators) on $A$, then F-IND ${ }^{2}$ is the class of all second order relations of the form $\psi(\bar{x}, \bar{Y}) \Leftrightarrow I_{\varphi}(\bar{a}, \bar{x}, \bar{Y})$, for some operative $\varphi(\bar{u}, \bar{x}, R, \bar{Y})$ in $F$ and constants $\bar{a}$ from $A$. As in [5] F-IND is the class of all relations on $A$ which are in F-IND ${ }^{2}$. We let $F^{\text {mon }}$ be the collection of all operative $\varphi(\bar{y}, R, \bar{Y})$ in $F$ which are monotone on $R$ and we put $\neg F=\{7 \varphi: \varphi \in F\}$. A collection of operators $F$ on $A$ is adequate if it contains all the $\Pi_{1}^{0}(C)$ second order relations, where $C$ is a coding scheme on $A$ and is closed under $\wedge, \vee, \exists^{A}$ and trivial combinatorial substitutions. Let $W F(S) \Leftrightarrow S$ be a well-founded relation on $A \Leftrightarrow \neg \exists a_{0} a_{1} a_{2} \cdots$ $\forall i\left(a_{i+1}, a_{i}\right) \in S$.

THEOREM 1. Let $F$ be an adequate collection of operators on an infinite set $A$. If $W F \in \neg F$ and $\neg F \subseteq F^{\text {mon }}$-IND ${ }^{2}$, then $F-I N D^{2}=F^{\text {mon }} \cdot I^{2} D^{2}$.

2. Elementary induction. Let $E L$ be the collection of all the elementary second order relations on a structure $A=\left\langle A, R_{1} \ldots R_{l}\right\rangle$ and let $E L^{+}$be the subcollection of $E L^{\text {mon }}$ consisting of all operative $\varphi(\bar{x}, R, \bar{Y})$ which are definable by positive in $R$ elementary formulas. One usually writes $E L^{+}-\mathrm{IND}^{2}=$ $\mathrm{IND}^{2}$ and $E L^{+} \cdot \mathrm{IND}=\mathrm{IND}$. Clearly $\mathrm{IND}^{2} \subset E L^{\mathrm{mon}}-\mathrm{IND}^{2} \subset E L-\mathrm{IND}^{2}$ and it is well known that $\mathrm{IND}^{2}$ is a tiny part of $E$ L-IND ${ }^{2}$ for (say) almost acceptable A's. By a basic result of Kleene and Spector for $\omega$ and Barwise-Gandy-Moschovakis in general (see $[4, \S 8 \mathrm{~A}]$ ), on every countable almost acceptable structure, $\mathrm{IND}^{2}=E L^{\text {mon }}-\mathrm{IND}^{2}\left(=\Pi_{1}^{1}\right)$. On the other hand, letting $W F^{n}(S) \Leftrightarrow S$ is a 2 n-ary relation on $A$ which is well founded (viewed as binary on $A^{n}$ ), we have

COROLLARY 1. Let $A$ be an infinite structure such that each $W F^{n}$ is elementary. Then $E L^{\text {mon }}-\mathrm{IND}^{2}=E L-\mathrm{IND}^{2}$.

AMS (MOS) subject classifications (1970). Primary 02 F27. 
A more detailed level-by-level version of Corollary 1 is the following, where we just write $\Sigma_{m}^{0}, \Pi_{m}^{0}$ instead of $\Sigma_{m}^{0}(C), \Pi_{m}^{0}(C)$, where $C$ is a hyperelementary coding scheme on $A$.

COROLlARY 2. Let $\mathrm{A}$ be an almost acceptable structure. If $m \geqslant 2$ and $W F \in \Pi_{m}^{0}$, then for all $n \geqslant m, \Sigma_{n}^{0}$ IND $^{2}=\left(\Sigma_{n}^{0}\right)^{\text {mon }}-\mathrm{IND}^{2}$.

So, for example, in the structure of analysis $\mathrm{R}$ this says that $\boldsymbol{\Sigma}_{n}^{\mathbf{1}}$ monotone operators on $R$ inductively define the same relations as arbitrary $\Sigma_{n}^{1}$ operators, when $n \geqslant 2$. Similarly for $\Sigma_{n}^{1}$. The following rather curious result can be also established by the methods used to prove Theorem 1 . If $A=\left\langle A, R_{1} \cdots R_{l}\right\rangle$ is a structure, by an elementary quantifier $Q$ on $A$ we understand a quantifier on $A$ which viewed as a second-order relation is elementary.

THEOREM 2. Let A be an acceptable structure in which WF is elementary. There is an elementary quantifier $Q$ on $A$ such that for every inductive relation $R$ on $A$, there is an inductive relation $R^{*}$ on $A$ such that $\neg R(\bar{x}) \Leftrightarrow Q y R^{*}(\bar{x}, y)$.

This should be compared with a result of Moschovakis [3] in higher type recursion, where "inductive" is replaced by "semirecursive in a total object of type $\geqslant 3$ " and $Q$ becomes the existential quantifier (on an appropriate space).

REMARKS. (i) We conjecture that in Theorem 1 (and correspondingly in Corollary 1 ) the hypothesis $W F \in \neg F$ can be weakened to $W F \in \neg\left(F^{\text {mon }}-I N D^{2}\right)$. (ii) In a direction opposite to that of Corollary 1 one has the following theorem of Nyberg (unpublished): Let $A$ be almost acceptable. If IND $\nsubseteq-\left(E L^{\mathrm{m}}{ }^{\text {on }}\right.$-IND), then $E L^{\text {mon }}$-IND $=$ IND. Thus for most structures occuring in practice, $E L^{\text {mon }}$-IND is either IND or EL-IND.

3. Further corollaries and applications to Spector classes. An immediate consequence of Theorem 1 is also the following result of Harrington and Moschovakis [2]. (Given a structure $A$ and a quantifier $Q$ on $A$ we abbreviate by $Q$-IND the class of second order relations which are positive $L^{A}(Q)$-inductive (see [4, p. 49]).

Corollary 3. (Harrington-Moschovakis [2]). Let $A$ be an almost acceptable structure and let $Q$ be a quantifier on $A$. If $F=\neg\left(Q-\mathrm{IND}^{2}\right)$, then $F$ $\mathrm{IND}^{2}=F^{\text {mon }}-\mathrm{IND}^{2}$.

This generalizes a result of Grilliot to the effect that over $\omega, \Sigma_{1}^{1}-\mathrm{IND}^{2}=$ $\left(\Sigma_{1}^{1}\right)^{\text {mon }}$-IND ${ }^{2}$. The original proof of Corollary 2 in [2] yields the stronger statement that for $F=7\left(Q-\mathrm{IND}^{2}\right), F-\mathrm{IND}^{2}=F^{\text {pos }}$-IND ${ }^{2}$ and also shows that F-IND ${ }^{2}=Q^{+}-\mathrm{IND}^{2}$, where $Q^{+}$is the next quantifier of $Q$ (see [1]). Turning now to Spector classes we can obtain the following, where the notions involved are explained in [5].

Theorem 3. Let $\Gamma$ be a Spector class on $A$, and let $F$ be a reasonable, 
nonmonotone class of operators on $A$ closed under $\exists^{A}$. If $W F \in \neg F$, then $\Gamma$ is F-compact iff $\Gamma$ is $F_{*}^{\text {on }}$-compact, where $F_{*}^{m o n}=\{\varphi(R): \varphi \in F, \varphi$ monotone $\}$. In particular if $F$ is typical, nonmonotone, $F^{\mathrm{mon}}$-IND is a Spector class iff $F^{\text {mon }}$-IND $=$ F-IND.

Further applications of the methods developed here to the theory of "second order" Spector classes as well as details and proofs of the results announced here will appear elsewhere.

\section{REFERENCES}

1. P. Aczel, Quantifiers, games and inductive definitions, Proc. Third Scandinavian Logic Sympos. (S. Kanger, Editor), North-Holland, Amsterdam; American Elsevier, New York, 1975, pp. 1-14. MR 51 \#5256.

2. L. A. Harrington and Y. N. Moschovakis, On positive induction vs. nonmonotone induction, Mimeographed notes, 1975.

3. Y. N. Moschovakis, Hyperanalytic predicates, Trans. Amer. Math. Soc., 129 (1967), 249-282. MR 38 \#308.

4. - Elementary induction on abstract structures, North-Holland, Amsterdam, 1974.

5. - On nonmonotone inductive definability, Fund. Math. 82 (1974), 39-83. MR 50 \#6853.

DEPARTMENT OF MATHEMATICS, UNIVERSITY OF CALIFORNIA, BERKELEY, BERKELEY, CALIFORNIA 94720

DEPARTMENT OF MATHEMATICS, CALIFORNIA INSTITUTE OF TECHNOLOGY, PASADENA, CALIFORNIA 91125 